\title{
Magnetic excitations and effects of magnetic fields on the spin-Peierls transition in $\mathrm{CuGeO}_{3}$
}

\author{
José Riera and Sergio Koval \\ Instituto de Física Rosario, Consejo Nacional de Investigaciones Científicas y Técnicas, \\ and Departamento de Física, Universidad Nacional de Rosario, \\ Avenida 27 de Febrero 210b, 2000-Rosario, Argentina.
}

(Received )

\begin{abstract}
We analyze the magnetic excitations of a spin-1/2 antiferromagnetic Heisenberg model with alternating nearest neighbor interactions and uniform second neighbor interactions recently proposed to describe the spin-Peierls transition in $\mathrm{CuGeO}_{3}$. We show that there is good agreement between the calculated excitation dispersion relation and the experimental one. We have also shown that this model reproduces satisfactorily the experimental results for the magnetization vs. magnetic field curve and its saturation value. The model proposed also reproduces qualitatively some features of the magnetic phase diagram of this compound and the overall behavior of the magnetic specific heat in the presence of applied magnetic fields.
\end{abstract}

PACS Numbers: 61.50.Em, 64.70.kb, 75.10.Jm, 75.40.Mg, 75.50.Ee

Typeset Using REVTEX 
Interest in low-dimensional quantum spin systems has been intensified in recent years by the observation of a rich variety of behaviors in new materials and by important theoretical results. [1.2] In particular, the spin-Peierls (SP) transition is one of the most attractive phenomena and it was first discovered and studied in organic compounds. [3] At the SP transition, the high-temperature phase with uniform spin-1/2 Heisenberg antiferromagnetic chains ("U phase") changes into the low-temperature phase with dimerized or alternating chains ("D phase"). In the dimerized phase the system is characterized by the presence of an energy gap (the "SP gap") between the singlet ground state and the first triplet state.

Recently the SP transition was observed in the inorganic material $\mathrm{CuGeO}_{3}$ by Hase, Terasaki and Ichinokura. [1] They showed that the magnetic susceptibility in $\mathrm{CuGeO}_{3}$ along all directions drop drastically at the transition temperature $\mathrm{T}_{\mathrm{SP}} \approx 14 \mathrm{~K}$. The SP nature of this transition has been confirmed by several experimental results. [5.6.6.8.89.10] The quasi one-dimensional (1D) nature of this compound is given by the presence of weakly coupled linear $\mathrm{Cu}^{2+}$ spin- $1 / 2$ chains along the $c$ direction. The transition is the result of the coupling between these chains and the three-dimensional phonons. [6, []

In order to characterize the SP phase in this compound, it is important to investigate its thermodynamic properties in the presence of magnetic fields because as it is well-known in organic SP materials they have characteristic behaviors below $\mathrm{T}_{\mathrm{SP}}$ and large magnetic fields. 11] The phase diagram of SP systems in the magnetic field and temperature plane, as determined experimentally from specific heat and ac susceptibility measurements, shows the uniform and dimerized phases at low magnetic fields, and another magnetic phase ("M phase") at high magnetic fields. In the absence of magnetic fields, the dimerization of the chains corresponds to a lattice distortion with wave vector $\pi$. For any $\mathrm{T}<\mathrm{T}_{\mathrm{SP}}$, the magnetic field above a critical value $\mathrm{H}_{\mathrm{c}}(\mathrm{T})$ favors a distortion at some other wave vector or another type of distortion. The M phase could then correspond to another conmensurate, discommensurate (or magnetic soliton) or inconmensurate phase. In the case of $\mathrm{CuGeO}_{3}$, this M phase has not yet been fully characterized.

Recently, in Ref. [12] ("RD" in the following) we attempted a description of the SP 
transition in $\mathrm{CuGeO}_{3}$ with a spin-1/2 1D Heisenberg antiferromagnetic model coupled to the three-dimensional phonons in the adiabatic approximation. The spin part of this model is defined by the Hamiltonian:

$$
\mathrm{H}_{\mathrm{s}}=\sum_{\mathrm{i}} \mathrm{J}_{\mathrm{i}} \mathbf{S}_{\mathrm{i}} \cdot \mathbf{S}_{\mathrm{i}+1}+\alpha \mathrm{J} \sum_{\mathrm{i}} \mathbf{S}_{\mathrm{i}} \cdot \mathbf{S}_{\mathrm{i}+2}
$$

where the index i runs over the lattice sites $(\mathrm{i}=1, \ldots, \mathrm{N}, \mathrm{N}$ : number of sites) with periodic boundary conditions. The exchange coupling constant $\mathrm{J}_{\mathrm{i}}=\mathrm{J}\left(1+\delta(-1)^{\mathrm{i}}\right)$ where $\delta=\delta(\mathrm{T})$ is a temperature dependent dimensionless quantity which gives the degree of dimerization. The model Eq. (11) has been studied so far in the limits $\alpha=0$ [13], and $\delta=0$. [14]

The parameters of the model were chosen so as to give a good fit of the experimental magnetic susceptibility and to reproduce the measured excitation gap at $\mathrm{T}=0$. [7, 8] The resulting parameters are: $\mathrm{J}=160 \mathrm{~K}, \alpha=0.36$ and $\delta(\mathrm{T}=0)=0.014$. As it is well-known, the 1D Heisenberg antiferromagnetic model with first and second neighbor interactions has a finite singlet-triplet gap for $\alpha>\alpha_{c} \approx 0.2412$. 114,15] Thus, the value of $\alpha=0.36$ implies that there is a finite gap even in the absence of dimerization. This spin gap was estimated in $\mathrm{RD}$ to be $\approx 0.015$ in units of $\mathrm{J}$, or $2.4 \mathrm{~K}$, much smaller than the smallest measured value for $\mathrm{CuGeO}_{3}$. [7] In a more recent study Castilla, Chakravarty and Emery [15] argued, from an analysis of the neutron scattering measurements of the superlattice peaks [7], that the value of $\alpha$ should be below the critical one. For this reason, they proposed the following set of parameters: $\mathrm{J}=150 \mathrm{~K}, \alpha=0.24$, and $\delta(\mathrm{T}=0)=0.030$. However, we think that this argument, based on the theory of Cross and Fisher [16] which uses a Luther-Peschel-type treatment of the spin Hamiltonian, is not conclusive. Moreover, we should notice that there is a rather large interchain coupling along the $b$ direction, $\mathrm{J}_{\mathrm{b}} / \mathrm{J} \approx 0.1[8]$ that could lead to a different effective $\alpha_{c}$.

The purpose of this work is to describe using the model proposed in RD other features of the SP phase in $\mathrm{CuGeO}_{3}$, in particular the nature of magnetic excitations and the behavior of various thermodynamic properties in the presence of an external magnetic field as have been revealed by an increasing amount of experimental results. We want to show also that 
the model and numerical procedure developed in RD could provide an alternative complete theory of the spin-Peierls transition and that it could be considered as an useful tool to analyze experimental data. These studies will be performed by using exact diagonalization techniques on finite chains. In these calculations we adopt the parameters given in RD which lead to a somewhat better fit to the experimental magnetic susceptibility as well as for the dispersion relation as we shall see below than the parameter set given in Ref. [15]. Most of the calculations were performed also for this latter parameter set and we shall discuss the main qualitative differences found.

We start our study with an analysis of magnetic excitations at zero temperature and in the absence of a magnetic field. For this study we have employed the Lanczos algorithm for lattices of up to 24 sites. We have also calculated the dynamical magnetic structure factor $\mathrm{S}(\mathrm{k}, \omega)$ for all the wave vectors $\mathrm{k}$. 17] This dynamical response has the largest intensity at $\mathrm{k}=0$ (corresponding to $\pi$ in the uniform lattice) and hence we shall concentrate our study to this momentum. The results for $\mathrm{S}(\mathrm{k}=0, \omega)$ are depicted in Fig. 1a for $\alpha=0.36$ and $\delta=0.014$. We also show the results for the nondimerized model for comparison. The first peak corresponds to the singlet-triplet excitation. This peak has the largest weight, in agreement with experimental results. [8] We also note other excitations at higher frequencies and with lower intensities. It is interesting to notice that the second peak in the nondimerized case is split by a finite $\delta$. The relative intensity of this second peak with respect to the first one remains approximately constant as $\mathrm{N}$ is increased. Similar results are obtained with the set of parameters of Ref. [15], although the second peak is located at slightly higher frequencies than for our set of parameters. In order to understand the origin of these excitations, let us examine them as a function of $\mathrm{k}$. The results for the $\mathrm{N}=24$ chain are shown in Fig. 1b together with recent neutron scattering results. [8] It can be seen that the parameter set of RD fits slightly better the experimental data than the parameter set of Ref. [15]. In Fig. 1c we show the excitation energy of various states as a function of $\mathrm{k}$ for the same chain. At $\mathrm{k}=0$ it is convenient to label each energy level by $\mathrm{E}_{\mathrm{i}}(\mathrm{S}, \mathrm{P})$ where $\mathrm{S}$ is the total spin, $\mathrm{P}(=+1,-1)$ is the quantum number associated to the reflection symmetry, and 
$\mathrm{i}=0$ (1) for the ground state (first excited state). For $\mathrm{N}=4 \mathrm{n}, \mathrm{n}$ integer, the singlet-triplet spin gap is $\Delta_{s t}=\mathrm{E}_{0}(1,-)-\mathrm{E}_{0}(0,+)$. For $\mathrm{N}=4 \mathrm{n}+2$, the values of $\mathrm{P}$ are reversed. The values of $\mathrm{P}$ quoted below correspond to the case $\mathrm{N}=4 \mathrm{n}$.

By studying the excitation spectra for several lattice sizes, we obtained that the position of the second peak in $S(\mathrm{k}=0, \omega)$ is $\omega_{1}=\mathrm{E}_{1}(1,-)-\mathrm{E}_{0}(0,+)$. The excited singlet state $\mathrm{E}_{1}(0,+)$ appears inside the singlet-triplet gap but this is just a finite size effect as can be seen in Fig.1d. This feature is absent for $\alpha=0.24$, i.e. $\mathrm{E}_{1}(0,+)>\mathrm{E}_{0}(1,-)$ for any lattice size. In Fig. 1d, we plot $\Delta_{s t}, \omega_{1}$ and $\omega_{2}=\mathrm{E}_{1}(0,+)-\mathrm{E}_{0}(0,+)$ as a function of $\mathrm{N}^{-1}$. The data do not seem to vary linearly with $\mathrm{N}^{-2}$ and there is some spread in the extrapolated values depending on the fitting function chosen. In Fig. 1d tentative extrapolations using the function $\omega=\omega_{\infty}+\mathrm{aN}^{-1}+\mathrm{bN}^{-2}$ are shown. It is apparent that in the bulk limit the singlet-triplet excitation becomes the lowest excitation in agreement with the case $\alpha=0.24$ and with experimental results. Besides, several trial extrapolations indicate that there would remain a finite gap between the first and second peaks of $\mathrm{S}(\mathrm{k}=0, \omega)$ in the thermodynamic limit for $\alpha=0.36$. The results are less conclusive for $\alpha=0.24$. Finally, we want to notice that the first excited triplet state has $\mathrm{P}=-1$ for $\alpha=0.36$ but $\mathrm{P}=+1$ for $\alpha=0.24$.

We turn now to the study of thermodynamic properties in the presence of an applied magnetic field $\mathrm{H}$ along the $c$ direction. In order to study the SP phase at finite temperature and in the presence of a magnetic field we minimize the free energy for the total Hamiltonian with respect to $\delta$ to obtain $\delta_{e q}(\mathrm{H}, \mathrm{T})$. The total Hamiltonian of the system consists of the spin part given by Eq. (四) with the addition of an elastic term $\sim \delta^{2}$ and the Zeeman term $\mathrm{g} \mu_{0} \mathrm{~S}^{z} \mathrm{H}$, where $\mathrm{g}$ is the Landé factor (we take $\mathrm{g}=2$ ), $\mu_{0}$ is the Bohr magneton and $\mathrm{S}^{\mathrm{z}}$ is the z-component of the total spin. The dimerization parameter $\delta_{e q}(\mathrm{H}, \mathrm{T})$ is, for a given $\mathrm{T}$, a decreasing function of $\mathrm{H}$ (Fig. 2a). Consequently, the spin gap and the critical temperature $\mathrm{T}_{\mathrm{SP}}(\mathrm{H})$ are also suppressed by the application of an external magnetic field, as discussed in several theoretical studies. [11, [16, 18] Once $\delta_{e q}(\mathrm{H}, \mathrm{T})$ is known, the computation of the thermodynamic properties we are interested in is straightforward. This procedure only requires the computation of the eigenvalues of the Hamiltonian (1) which is readily done by 
using the Householder algorithm for chains of up to 14 sites.

Recently the magnetization curve was measured in the presence of ultra-high magnetic fields up to $\approx 500 \mathrm{~T}$. [19] The measurement of the magnetization and its saturation can be used to determine the values of the antiferromagnetic exchange interactions for an adopted model Hamiltonian. Nojiri et al [19] noticed that the experimental magnetization curve shows good agreement with the theoretical curve obtained for the spin-1/2 uniform Heisenberg antiferromagnetic chain with nearest-neighbor coupling $\mathrm{J}=183 \mathrm{~K}$. However, as discussed in RD, this model does not satisfactorily reproduce the magnetic susceptibility data. We plot in Fig. 2b the magnetization $\mathrm{M}$ versus applied magnetic field $\mathrm{H}$ obtained with the model proposed in $\mathrm{RD}$ for $\mathrm{N}=14$ site chain at $\mathrm{T}=5$ and $8 \mathrm{~K}$. We also include the experimental results corresponding to $\mathrm{T}=6$ and $10 \mathrm{~K}$. [19] Our numerical curves present the typical steplike structure due to the finite lattices involved. We observe an overall good agreement of the theoretical curve compared to the experiment results. The temperature dependence of our results is somewhat weaker than the experimental one, specially for high magnetic fields where presumably the three-dimensional effects are more important. We point out here that without further parameters to adjust in our model, we have also obtained the value of the saturation field $(\approx 253 \mathrm{~T})$ in reasonable agrement with the experimental result. We obtain very similar results with the parameters of Ref. [15.

The knowledge of $\delta_{e q}(\mathrm{H}, \mathrm{T})$ enables us to discuss some features of the magnetic phase diagram of $\mathrm{CuGeO}_{3}$. This magnetic phase diagram has been partially determined experimentally by measuring the magnetization in magnetic fields up to $25 \mathrm{~T}$, [5] and it presents the three phases characteristic of a SP system. Hase et al. [5] showed that as the applied magnetic field is increased, at temperatures below $\mathrm{T}_{\mathrm{SP}}(0)$, there is a nonlinear increase of the magnetization above a certain $\mathrm{H}_{\mathrm{c}}(\mathrm{T})$. [22 This nonlinear increase, together with the presence of hysteresis, indicates that there is a first order transition between the dimerized phase and the high-field $\mathrm{M}$ phase for $\mathrm{T} \leq 10 \mathrm{~K}$. The hysteresis disappears above this temperature and the D-U phase boundary becomes a second order one. This boundary is determined in our microscopic theory by the condition $\delta_{e q}(\mathrm{H}, \mathrm{T})=0$. The results for $\mathrm{N}=12,14$ and 16 
site chains [21] using our parameters set are plotted in Fig. 3. We also show for comparison the analytical results obtained by Cross [23] using the theory developed in Ref. [16]. The most important assumption of this theory is that $\mathrm{T}_{\mathrm{SP}}(0) \ll \mathrm{J}$, moderately satisfied in this compound. For low magnetic fields, our results closely agree with the curve calculated by Cross corresponding to the D-U transition (dashed line) and with experimental results. [5.,20]

For higher magnetic fields, the theory of Ref. [23] predicts a transition between the highfield magnetic (assumed incommensurate) and the uniform phases, which is also shown in Fig. 3 (full line). There are still no experimental results for $\mathrm{CuGeO}_{3}$ corresponding to this $\mathrm{M}-\mathrm{U}$ transition. In order to study the incommensurability of the $\mathrm{M}$ phase and the transitions D-M and M-U, the nearest neighbor coupling constants in our model should be generalized as $\mathrm{J}_{\mathrm{i}}=\mathrm{J}(1+\delta \cos (\mathrm{qi}))$ where $\mathrm{q}=\mathrm{q}(\mathrm{H})$ is the component of the phonon wave vector parallel to the chain direction. The dimerization studied so far corresponds to the commensurate value $\mathrm{q}=\pi$. In the presence of a magnetic field the value of $\mathrm{q}$ is the one that minimizes the free energy of the system. At present there are important numerical limitations to accomplish such a program. Moreover, it should be noticed that the interchain exchange interactions have stronger effects in the M phase. [24] Although we have not attempted an extrapolation to the bulk limit, the curves obtained with our model in the high-field region should converge as the chain size is increased to an upper bound to the D-M boundary. The determination of the first-order nature of the D-M phase transition is also out of the scope of this work because of the limitation in the numerically accessible chain sizes.

Finally, we study the magnetic specific heat $\mathrm{C}_{\mathrm{m}}$ in the presence of a magnetic field. The results for $\mathrm{C}_{\mathrm{m}} / \mathrm{T}$ obtained for the $\mathrm{N}=14$ site chain as a function of $\mathrm{T} / \mathrm{T}_{\mathrm{SP}}$ for $\mathrm{H}=0,5$ and $10 \mathrm{~T}$ are shown in Fig. 4. Experimentally, it has been found that the specific heat shows a sharp anomaly at $\mathrm{T}_{\mathrm{SP}}$ due to the $\mathrm{SP}$ transition 25,26]. This peak is clearly seen in our results. Consistently with the results shown in Fig. 2b, the position of the peak corresponding to the SP transition decreases as the magnetic field is increased and the low temperature part of the curve moves upward. These changes of $\mathrm{C}_{\mathrm{m}} / \mathrm{T}$ with magnetic field are also in qualitative agreement with the experimental data from Ref. [26]. The differences 
in shape between the theoretical and experimental curves are presumably also a finite size effect.

In summary, we have analyzed magnetic excitations of the spin- $1 / 2$ antiferromagnetic Heisenberg model with alternating nearest neighbor interactions and uniform second neighbor interactions proposed in $\mathrm{RD}$ to describe the spin-Peierls transition in $\mathrm{CuGeO}_{3}$. These results are among the first reported for this very complicated model and they could be relevant for future neutron scattering experiments. There is good agreement between the calculated excitation dispersion relation and the experimental one. We have also shown that this model reproduces satisfactorily the experimental results for the magnetization vs. magnetic field curve and its saturation value. The model proposed also reproduces qualitatively some features of the magnetic phase diagram of this compound and the overall behavior of the magnetic specific heat in the presence of applied magnetic fields.

We acknowledge H. Nojiri for sending us their results for M vs. H prior publication. S. K. acknowledge helpful discussions with A. Dobry and A. Greco during the early stages of this work. 


\section{REFERENCES}

[1] F. D. M. Haldane, Phys. Rev. Lett. 50, 1153 (1983).

[2] E. Dagotto, J. Riera, and D. J. Scalapino, Phys. Rev. B 45, 5744 (1992).

[3] For a review, see J. W. Bray, L. V. Interrante, I. S. Jacobs, and J. C. Bonner, in Extended linear chain compounds, edited by J. S. Miller, (Plenun, New York 1983), Vol 3, pp 353415.

[4] M. Hase, I. Terasaki and K. Uchinokura, Phys. Rev. Lett. 70, 3651 (1993).

[5] M. Hase et al., Phys. Rev. B 48, 9616 (1993).

[6] J. E. Lorenzo et al., Phys. Rev. B 50, 1278 (1994); K. Hirota et al., Phys. Rev. Lett. 73, 736 (1994).

[7] Q. J. Harris et al., Phys. Rev. B 50, 12606 (1994).

[8] M. Nishi, O. Fujita and J. Akimitsu, Phys. Rev. B 506508 (1994).

[9] T. M. Brill et al., Phys. Rev. Lett. 73, 1545 (1994).

[10] J. P. Pouget et al., Phys. Rev. Lett. 72, 4037 (1994).

[11] J. C. Bonner, J. A. Northby, I. S. Jacobs and L. V. Interrante, Phys. Rev. B 35, 1791 (1987).

[12] J. Riera and A. Dobry, Phys. Rev. B 51, 16098 (1995).

[13] J. C. Bonner et al., Phys. Rev. B 27, 248 (1983); M. Azzouz and C. Bourbonnais, preprint (1995).

[14] K. Okamoto and K. Nomura, Phys. Lett. A 169, 433 (1992); and references therein.

[15] G. Castilla, S. Chakravarty, and V. J. Emery, preprint (1995).

[16] M. C. Cross and D. S. Fisher, Phys. Rev. B 19, 402 (1979). 
[17] A study of $\mathrm{S}(\mathrm{k}, \omega)$ for all $\mathrm{k}$ and using the parameters of Ref. [15 was recently reported by S. Haas and E. Dagotto, preprint (1995).

[18] L. N. Bulaevskii, A. I. Buzdin and D. I. Khomski, Solid State Comm. 27, 5 (1978).

[19] H. Nojiri et al., preprint (1995).

[20] H. Hori et al., J. Phys. Soc. Jpn. 63, 18 (1994).

[21] The data corresponding to $\mathrm{N}=16$ are the result of an approximate calculation using the Lanczos algorithm. It is assumed that the use of translational symmetries breaks the level degeneracy.

[22] See also H.Ohta et al., J. Phys. Soc. Jpn. 63, 2870 (1994).

[23] M. C. Cross, Phys. Rev. B 20, 4606 (1979).

[24] S. Inagaki and H. Fukuyama, J. Phys. Soc. Jpn. 52, 877 (1983).

[25] H. Kuroe et al., J. Phys. Soc. Jpn. 63, 365 (1994); S. Sahling et al., Solid State Comm. 92, 423 (1994).

[26] S. B. Oseroff et al., Phys. Rev. Lett. 74, 1450 (1995). 


\section{FIGURES}

FIG. 1. a) Spin dynamical structure factor $\mathrm{S}(\mathrm{k}=0, \omega)$ for $\mathrm{N}=20$ with $\alpha=0.36$. The solid (dashed) line corresponds to $\delta=0.014(\delta=0)$. b) Excitation dispersion for the $\mathrm{N}=24$ chain. The solid line corresponds to $\mathrm{J}=160 \mathrm{~K}, \alpha=0.36$ and $\delta=0.014$ and the dashed line to $\mathrm{J}=150 \mathrm{~K}$, $\alpha=0.24$ and $\delta=0.030$. The experimental data (open circles) is taken from Ref. [8]. c) Excitation dispersion of various states for the $\mathrm{N}=24$ chain with $\mathrm{J}=160 \mathrm{~K}, \alpha=0.36$ and $\delta=0.014$. The two lowest singlet (triplet) states are indicated with squares (circles). d) $\Delta_{s t}$ (solid circles), $\omega_{1}$ (squares) and $\omega_{2}$ (open circles), defined in the text, versus $\mathrm{N}^{-1}$. The dotted lines are quadratic fits in $\mathrm{N}^{-1}$.

FIG. 2. a) The reduced dimerization constant $\delta(\mathrm{H})$ versus the applied magnetic field $\mathrm{H}$ at $\mathrm{T}=3 \mathrm{~K}$ (circles), $5 \mathrm{~K}$ (squares), $7 \mathrm{~K}$ (diamonds) and $9 \mathrm{~K}$ (triangles). b) The magnetization $\mathrm{M}$ as a function of magnetic field at different temperatures. The theoretical results for $\mathrm{N}=14$ are plotted with lines and the experimental data from Ref. [19] with symbols.

FIG. 3. Magnetic phase diagram of the $\mathrm{SP}$ system $\mathrm{CuGeO}_{3}$. Open circles, solid squares and open diamonds correspond to $\delta_{e q}(\mathrm{~T}, \mathrm{H})=0$ for $\mathrm{N}=12,14$ and 16 sites respectively. We also show the experimental data from Ref. [5] (crosses). The results obtained by Cross [23] are plotted with solid and dashed lines.

FIG. 4. Magnetic specific heat as a function of temperature, for different values of the applied magnetic field $\mathrm{H}$. 

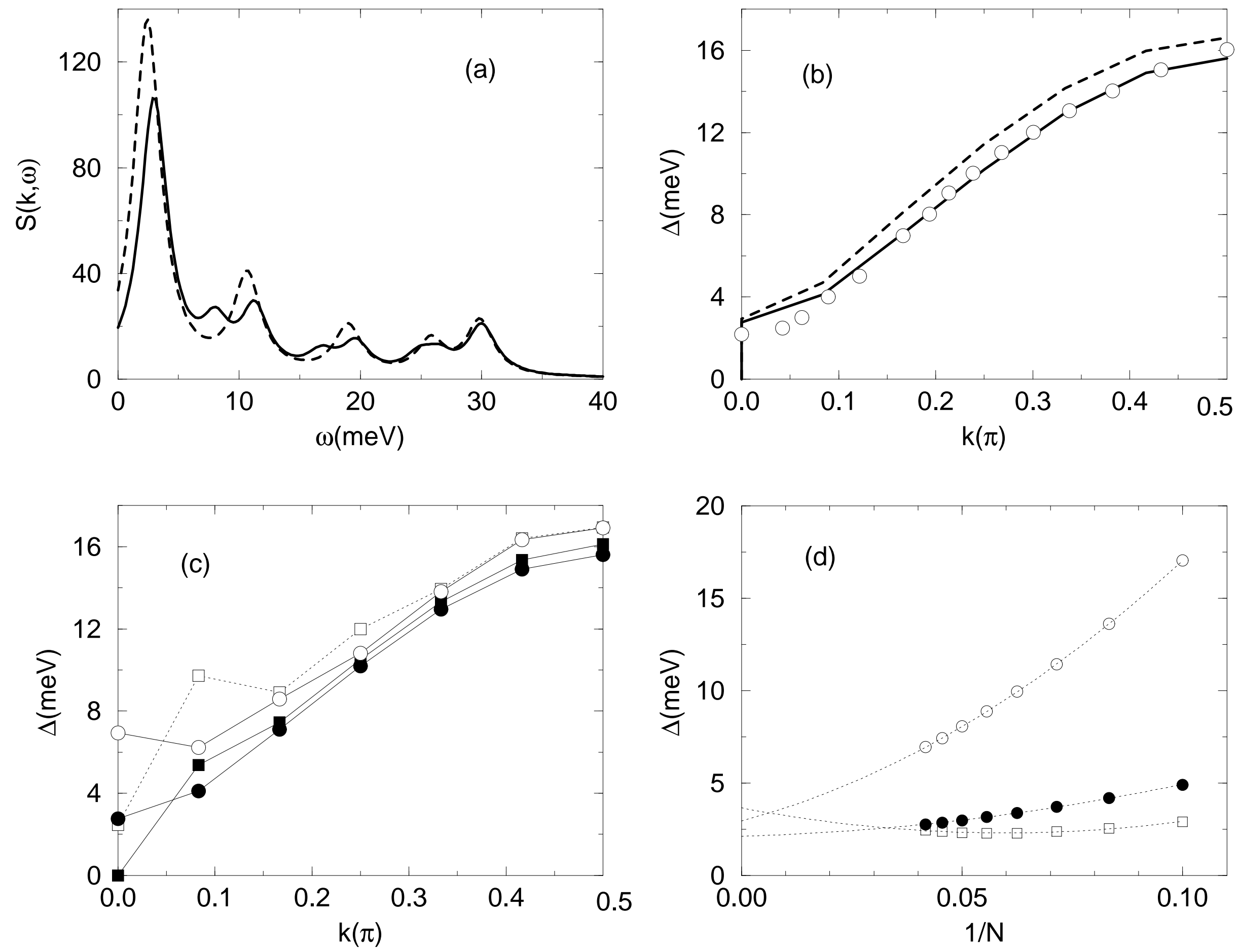

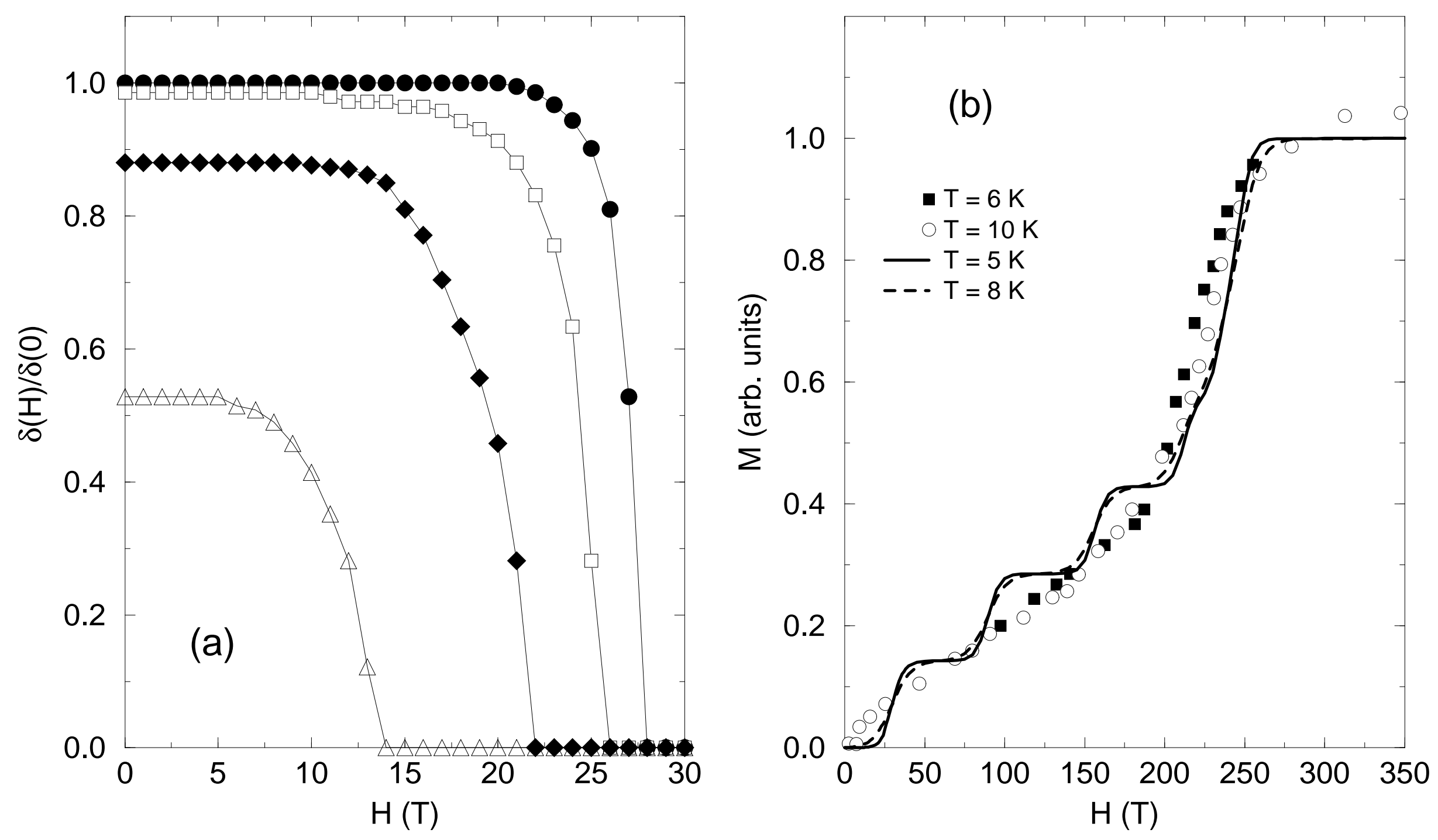


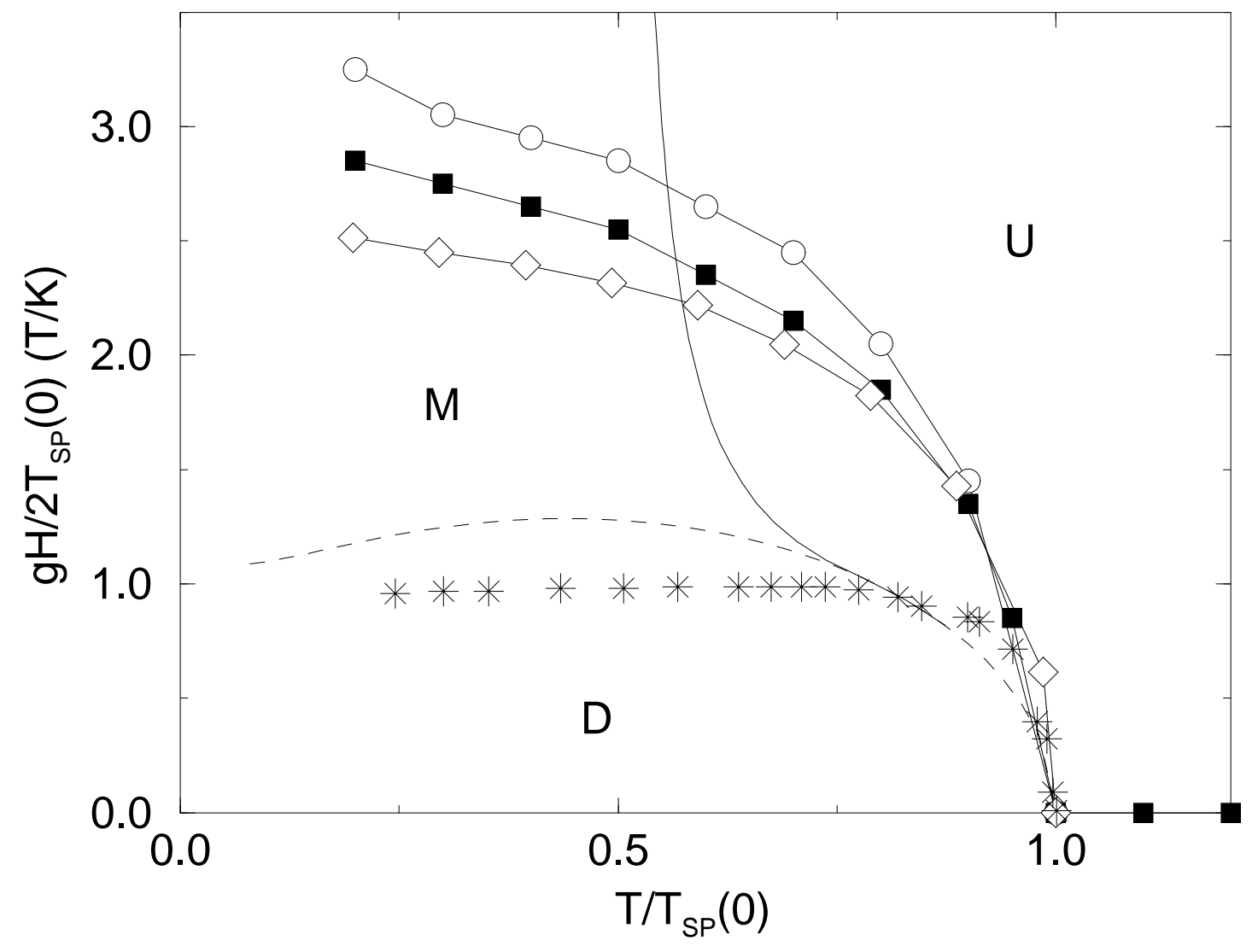




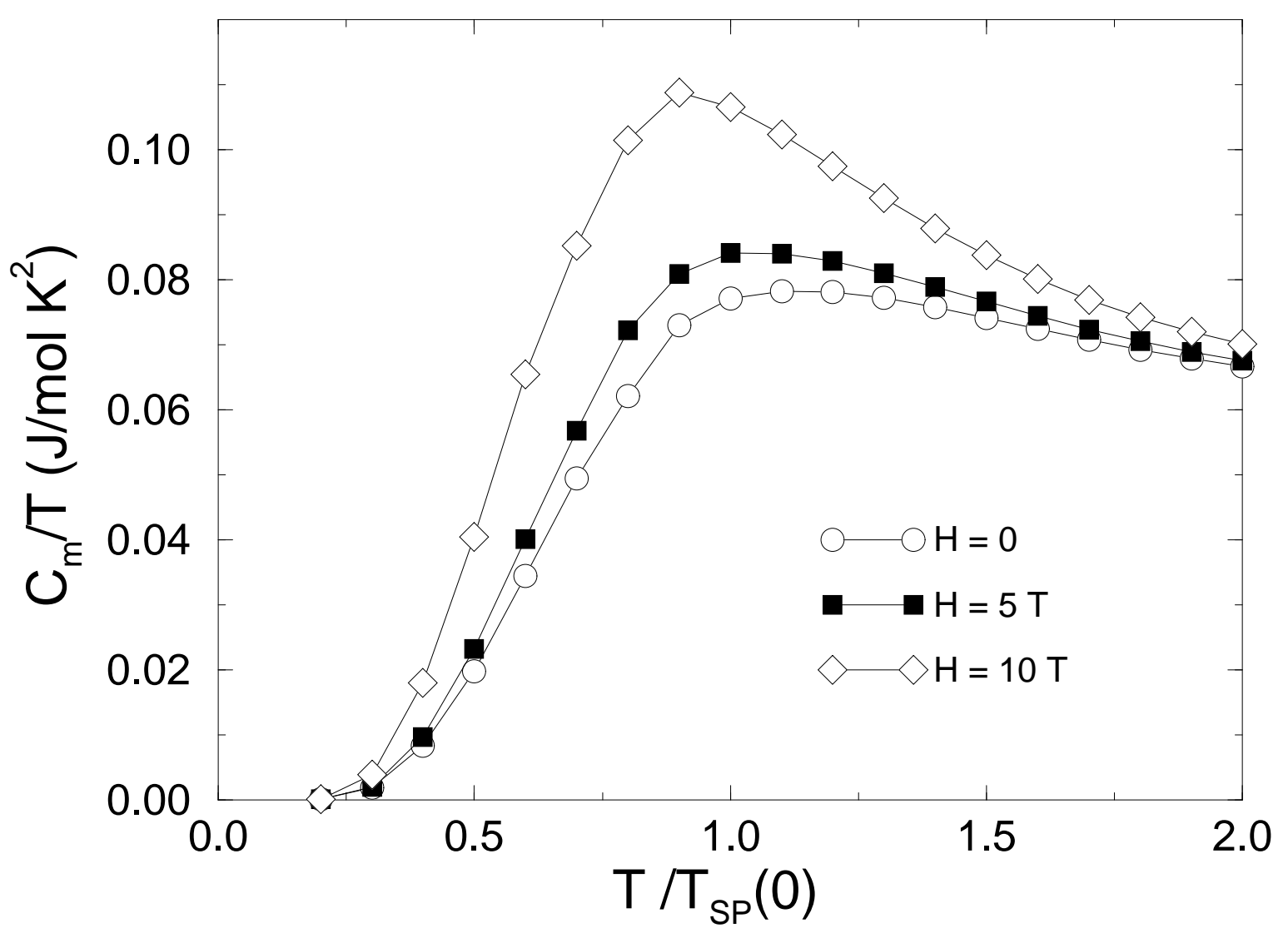

